\title{
The effects of EBL inhomogeneity on the gamma-gamma absorption of VHE gamma-rays
}

\author{
Hassan Abdalla * \\ Centre for Space Research, North-West University, Potchefstroom 2520, South Africa \\ Department of Astronomy and Meteorology, Omdurman Islamic University, Omdurman 382, \\ Sudan \\ E-mail: hassanahh@gmail.com
}

\section{Markus Böttcher}

Centre for Space Research, North-West University, Potchefstroom 2520, South Africa

Astrophysical Institute, Department of Physics and Astronomy, Ohio University, Athens, $\mathrm{OH}$

45701, USA

E-mail: Markus.Bottcher@nwu.ac.za

\begin{abstract}
Very High Energy Gamma Rays (VHE; more than $100 \mathrm{GeV}$ ) from Cosmological Gamma Ray Sources such as blazars can be absorbed by the Extragalactic Background light (EBL), which leads to a high-energy cut-off in blazar spectral energy distributions. However, recent observations of distant gamma ray sources suggest that the universe is more transparent to VHE gamma rays than expected from our current knowledge of a homogeneous EBL. One of the possible solutions is the hypothesis that a reduced EBL opacity results from inhomogeneities of the EBL density in particular if the line of sight to a blazar is passing through large voids in intergalactic space. We have evaluated the inhomogeneous and anisotropic EBL density and resulting gammagamma opacity in such a case. We find that even a sizeable void $R \lesssim 1 h^{-1}$ Gpc or many typical voids $R \lesssim 100 h^{-1}$ Mpc located along the line of sight to a distant blazar leads to a reduction of the EBL opacity only around $15 \%$. EBL inhomogeneities are not expected to reduce the EBL gamma-gamma opacity significantly, and alternative solutions to the problem of hard VHE spectra of blazars may be required.
\end{abstract}

4th Annual Conference on High Energy Astrophysics in Southern Africa

25-27 August, 2016

Cape Town, South Africa

${ }^{*}$ Speaker. 


\section{Introduction}

Very High Energetic Gamma rays from distant blazars can be annihilated due to $\gamma \gamma$ absorption by low-energy intergalactic photons. The importance of this process for high-energy astrophysics was first pointed out by [1]. Currently the study of $\gamma \gamma$ absorption of VHE photons from highredshift sources is one of the hot topics in high energy astrophysics due to their potential to study the cluster environments of blazars [2] and estimate cosmological parameters [3]. However, The EBL is very difficult to be measured accurately due to foreground emissions [4]. Studies of the EBL therefore focus on the predicted $\gamma \gamma$ absorption imprints and employ a variety of theoretical and empirical methods $[5,6,7,8,9,10,11,12]$. Recent observations of distant $(z \gtrsim 0.5) \gamma$-ray blazars have been interpreted by some authors $[13,14]$ that the universe may be more transparent to very high energy $\gamma$-rays than expected based on all existing EBL models. Possible solutions include the hypothesis that the EBL density is generally lower than expected from current models [15]; the existence of exotic Axion Like Particles (ALPs) [16]; extragalactic Ultra-high-Energy Cosmic Rays (UHECRs) [17]; and EBL inhomogeneities [18, 19, 20]. Based on a detailed calculation of the opacity due to an inhomogeneous EBL, including cosmic voids of typical sizes between the observer and a distant blazar, we have shown that for realistic void sizes of $R \lesssim 100 h^{-1} \mathrm{Mpc}$, the EBL energy density even at the center of the void is reduced by less than $10 \%$. Even if the void is located right in front of the background $\gamma$-ray source, the $\gamma \gamma$ opacity is reduced by typically less than $1 \%$. More details regarding to this work can be found in [20]. In the current work we consider an unrealistic extreme void or an equivalent accumulation of about 10 voids of typical sizes, distributed along the line of sight, to investigate whether this could explain the spectral hardening feature in the VHE spectra of some blazars such as PKS 1424+24.

\section{EBL in the Presence of a Cosmic Void}

Our calculations of the inhomogeneous EBL are based on a modified version of the formalism presented in [9], considering only the direct starlight. The effect of re-processing of starlight by dust has been included in [10] and leads to an additional EBL component in the mid- to far infrared, which is neglected here. Since dust re-processing is a local effect, it will be affected by cosmic inhomogeneities in the same way as the direct starlight contribution considered here.

For the purpose of a generic study of the effects of cosmic voids along the line of sight to a blazar, we assume that a spherical cosmic void is located with its center at redshift $z_{v}$ and raduis $R$ between the observer and a $\gamma$-ray source at redshift $z_{s}$. The geometry is illustrated in Figure 1 . We calculate the angle- and photon-energy-dependent EBL energy density at each point between the observer at redshift $\mathrm{O}$ and the source by using co-moving cordinates, converting redshifts $z$ to distances $l(z)$. The cosmic void is represented by setting the star formation rate to 0 within the volume of the void.

We start out by modifying the expression from [9] for the differential photon number density of the EBL at a given redshift $z$, based on the direct contribution from stars throughout cosmic history. For more details see [20].

Figure 2 illustrates the effect of the void on the differential EBL energy density as a function of distance along the line of sight to the $\gamma$-ray source, for one representative EBL photon energy, 


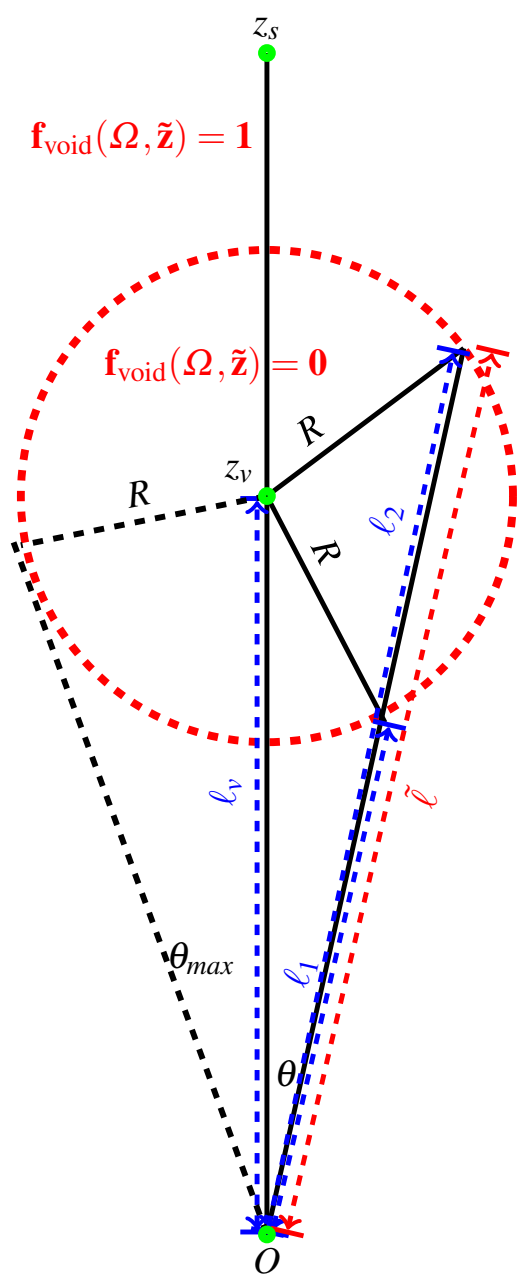

Figure 1: Illustration of the geometry of an underdense region between the observer at redshift $O$ and source at redshift $z_{s}$. We assume that the underdense region has a radius $R$ and the redshift at the center of the underdense region is $z_{v}$

in near-UV ( $\varepsilon=8 \mathrm{eV}$ ). The figure illustrates that the maximum effect (at the center of the void) is approximately proportional to the size of the void, but does not exceed $\sim 7 \%$ in the case of the $R=100 h^{-1}$ Mpc void. Also, we can notice that, when we double the void radius, the relativie EBL-energy-density-deficit will double. We can therefore conclude that the effect of a single large void with radius $R$ is approximately equivalent to the effect of a number of smaller voids with radii adding up to $\mathrm{R}$. 


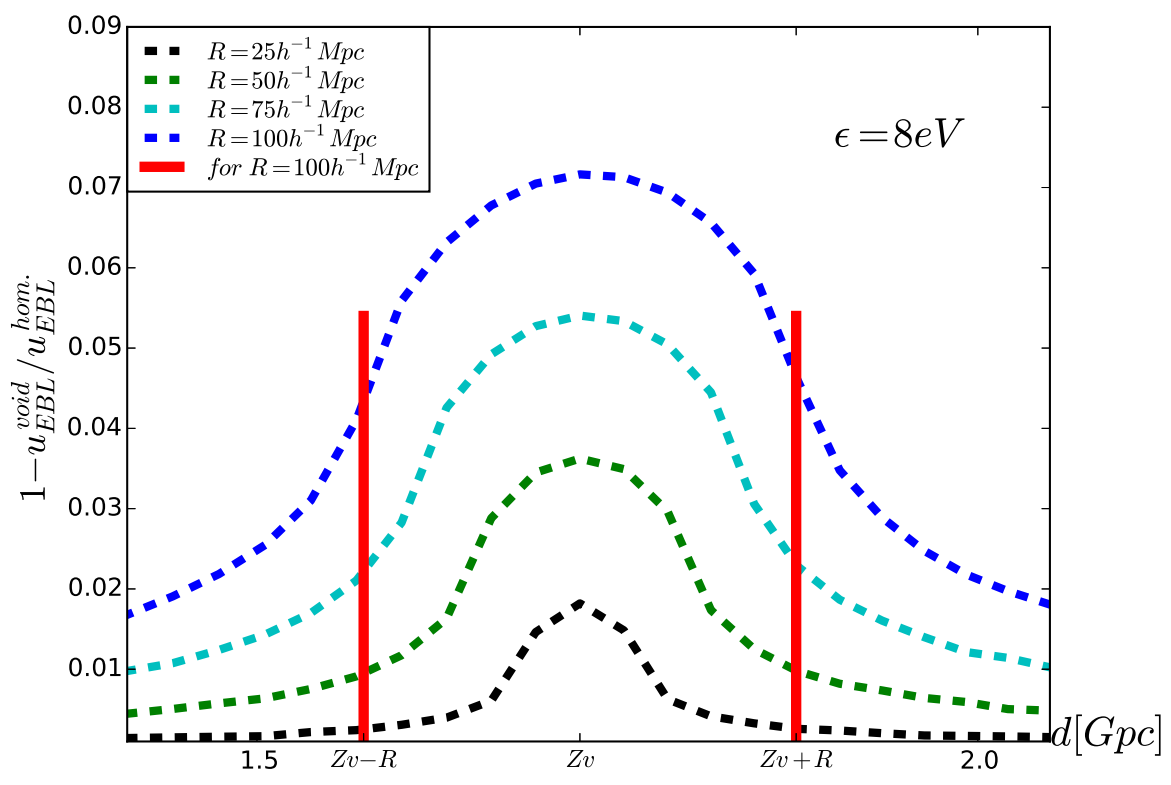

Figure 2: Relativie EBL energy density deficit due to the presence of the void for the same cases as in the top panels. The red vertical lines indicate the boundaries of the void for the $R=100 h^{-1} \mathrm{Mpc}$ case [20].

\section{Gamma-gamma Absorption in the presence of a void}

To study the influence of a cosmic void along the line of sight to a distant VHE- $\gamma$-rays source on the EBL energy density and resulting $\gamma \gamma$ opacity, we used the formalism described in the previous section (with the geometry illustrated in Figure 1). We consider spherical unrealistically large void sizes $R \lesssim 1 h^{-1}$ Gpc or equivalently around ten realistic void sizes $R \lesssim 100 h^{-1}$ Mpc distributed along or very close to the line of sight to a distant blazar. The center of the void is assumed to be located at a redshift of $z_{v}=0.3$, considering a source located at redshift $z_{s} \geq 0.6$.

Figure 3 (left panel) compares the EBL energy density spectrum for the case of a void (dashed lines), compared to the homogeneous case (solid lines). The right panel of Figure 3 shows the fractional difference between the homogeneous and the inhomogeneous case as a function of photon energy for various redshifts along the line of sight. In the left panel of Figure 4, we compare the resulting gamma-gamma opacities for the case of a voide (dashed lines) and the homogeneous case (solid lines), and the right panel shows the gamma-gamma optical depth deficit due to the presence of the voids for the same two cases as in the left panel.

We can notice that for even unrealistically large void sizes or an equivalent accumulation of about 10 voids of typical sizes, most of them distributed excactly along-the line of sight with the remaining ones located very close to the line of sight, the EBL energy density even at the center of the void is reduced by around $35 \%$ and the resulting maximum $\gamma \gamma$ opacity reduced by around $15 \%$. This is because even if the star-formation rate is set to zero within the void, the EBL density within the void is still substantial due to the contributions from the rest of the Universe outside the void. 

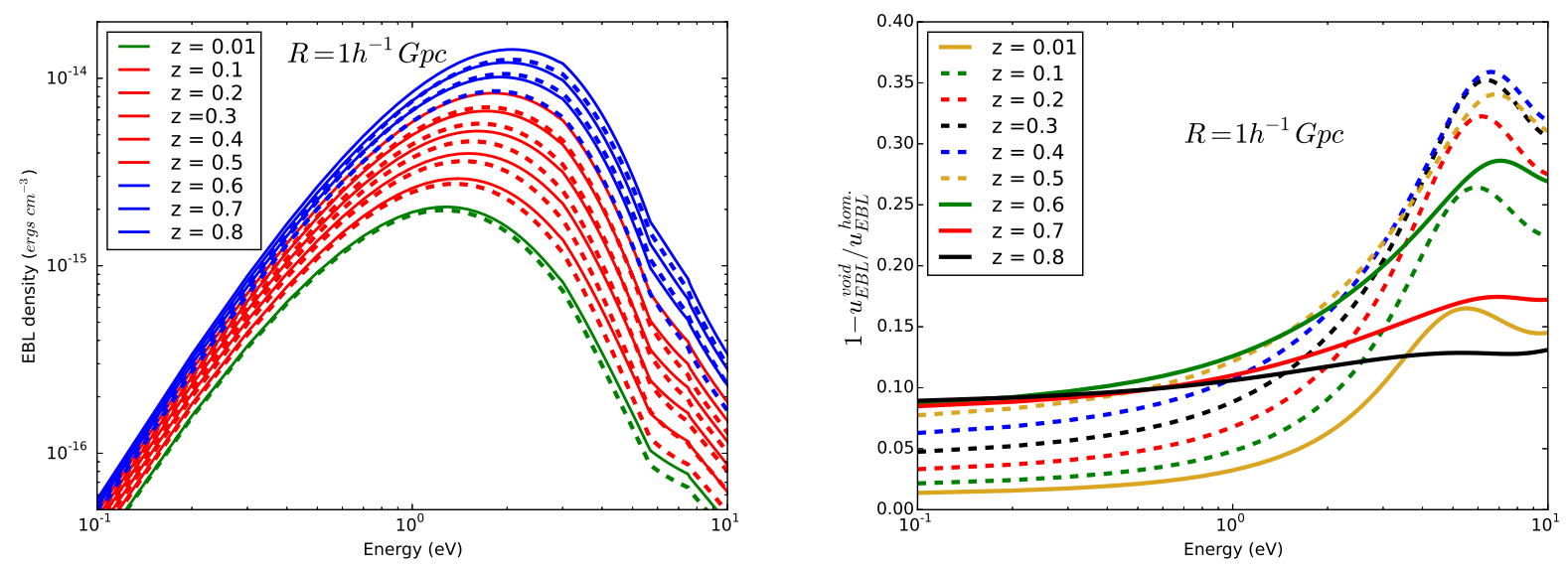

Figure 3: Left panel: Angle-averaged EBL photon energy density spectra for a homogeneous EBL (solid lines) and in the presence of a spherical cosmic void (dahed lines) with its center at redshift $z_{v}=0.3$ and with radius $R=1 h^{-1} \mathrm{Gpc}$. Green curves indicate locations in front of the void, red within the void, and blue behind the void. Right panel: Relative deficit of the EBL energy density due to the void, dash lines and solid lines represents the effect of void to the EBL-energy-density inside and outside the void respectively.
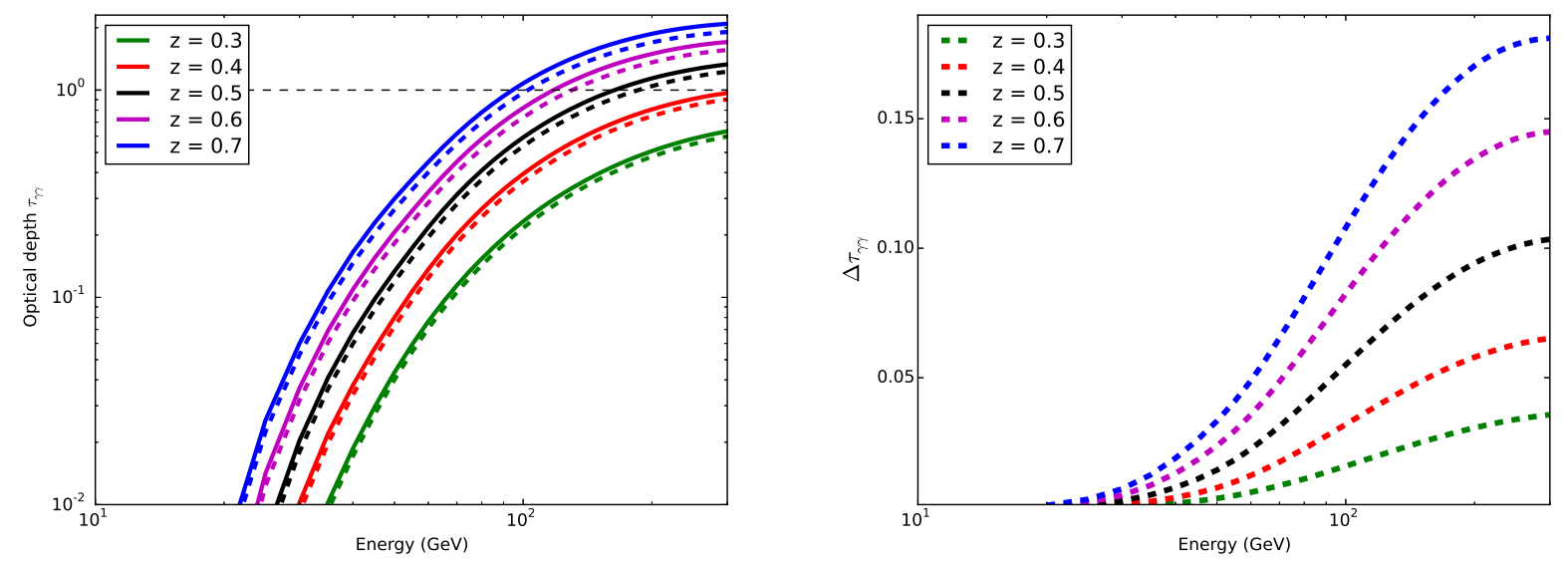

Figure 4: Left panels: EBL $\gamma \gamma$ optical depth as a function of $\gamma$-ray photon-energy in the presence of a void (dashed), compared to the homogeneous case (solid), for the same example voids as illustrated in Figure 3. Right panels: $\gamma \gamma$ optical depth deficit due to the presence of the voids for the same two cases as in the left panel.

\section{Summary and Conclusions}

We have presented detailed calculations of the effect of cosmic inhomogeneities on the EBL and the resulting $\gamma \gamma$ opacity for VHE $\gamma$-ray photons from sources at cosmological distances. Specifically, we have considered the presence of a large cosmic void, which, for simplicity, we have represented as a spherical region in which the local star formation rate is zero. We have shown that for unrealistically large void sizes $R \lesssim 1 h^{-1} \mathrm{Gpc}$ or equivalently around ten realistic void sizes $R \lesssim 100 h^{-1}$ Mpc distributed along or very close to the line of sight to a distant blazar, the EBL 
energy density even at the center of the void is reduced by around $35 \%$. Even if the void is located right in front of the background $\gamma$-ray source, the $\gamma \gamma$ opacity is reduced by typically less than $15 \%$. Specifically, we found that even with an alignment of about 10 realistic voids towards a blazar at $\mathrm{z}=0.6$ (such as PKS 1424+240), gamma-rays with energies above $130 \mathrm{GeV}$ are expected to be heavily attenuated. In order to suppress the gamma-gamma opacity sufficiently up to energies of $300 \mathrm{GeV}$, one would need more than 40 voids of typical sizes $R \lesssim 100 h^{-1}$ Mpc located exactly along or very close to the line of sight. This is clearly unrealistic and in conflict with observational constraints.

As with our current knowledge of the EBL, the spectral hardening feature in PKS 1424+240 and a few other VHE $\gamma$-ray blazars remains even when considering possible EBL inhomogeneities. Thus, other explanations for such hardening must be invoked. One possibility is that this hardening is, in fact, a real, intrinsic feature of the $\gamma$-ray spectra of these blazars, possibly due to a pion-production induced cascade component in a hadronic blazar model scenario [21, 22]. If such a spectral hardening is not intrinsic to the source, more exotic explanations, such as ALPs or a cosmic-ray induced secondary radiation component, would need to be invoked.

\section{Acknowledgments}

The work of M.B. is supported through the South African Research Chair Initiative of the National Research Foundation ${ }^{1}$ and the Department of Science and Technology of South Africa, under SARChI Chair grant No. 64789.

\section{References}

[1] Nikishov, A. I. 1962, Sov. Phys. JETP, 14, 393

[2] Sushch, I. \& Böttcher, M. 2015, A \& A, 573, A47

[3] Biteau A. \& Williams D. A. 2015, ApJ, 812, 1

[4] Hauser, M. G. \& Dwek, E. 2001, ARA\&A,, 39, 249

[5] Stecker, F. W. 1969, ApJ, 157, 507

[6] Stecker, F. W., de Jager, O. C., \& Salamon, M. H., 1992, ApJ, 390, L49

[7] Aharonian F. et al. 2006, Nature, 440, 1018

[8] Franceschini, A., Rodigheiro, G., \& Vaccari, M., 2008, A\&A, 487, 837

[9] Razzaque S., Demer, C. D., \& Finke, J. D., 2009, ApJ, 697, 483

[10] Finke, J. D., Razzaque, S., \& Dermer, C. D., 2010, ApJ, 712, 238

[11] Dominguez, A., et a., 2011a, MNRAS, 410, 2556

[12] Gilmore, R. C., Sommerville, R. S., Primack, J. R., \& Dominguez, A., 2012, MNRAS, 422, 3189

[13] MAGIC Collaboration: Albert, J., et al., 2008, Science, 320, 1752

\footnotetext{
${ }^{1}$ Any opinion, finding and conclusion or recommendation expressed in this material is that of the authors and the NRF does not accept any liability in this regard.
} 
[14] Archambault S. et al. 2014, ApJ, 785, L16

[15] Furniss A., et al., 2013, ApJ, 768, L31

[16] Dominguez, A., Sánchez-Conde, M. A., \& Prada, F., 2011b, J. Cosmol. Astropart. Phys., 11, 020

[17] Essey W. \& Kusenko A. 2010, ApJ, 33, 81

[18] Furniss A., Stutter, P. M., Primack, J. R., \& Dominguez, A., 2015, MNRAS, 446, 2267

[19] Kudoda, A. M. \& Faltenbacher, A., 2016, PoS, HEASA2015, 20

[20] Abdalla, H. \& Böttcher, M., (2016, ApJ, submitted).

[21] Böttcher, M., Reimer, A., Sweeney, K., \& Prakash, A., 2013, ApJ, 768, 54

[22] Cerruti, M., Zech, A., Boisson, C., \& Inoue, S., 2015, MNRAS, 448, 910 